EPiC Series in Computing
Volume 63, 2019, Pages 80-89

\title{
A Strategy to Evaluate and Measure the Degree of Openness of Cloud Services Environment
}

\author{
Narayan Debnath ${ }^{1}$, Mario Peralta ${ }^{2}$, Carlos $\mathrm{Salgado}^{2}$, Lorena Baigorria ${ }^{2}$, \\ Germán Montejano ${ }^{2}$, Daniel Riesco ${ }^{2}$ and Emdad Ahmed ${ }^{3}$ \\ ${ }^{1}$ International Society for Computers and Their Applications (ISCA), \\ Winona, MN 55987, USA \\ ndebnathC@gmail.com \\ ${ }^{2}$ Departamento de Informática Facultad de Ciencias Físico-Matemáticas y \\ Naturales Universidad Nacional de San Luis, Ejército de los Andes 950. San Luis, C.P. 5700, \\ Argentina \\ [mperalta, csalgado, flbaigor, gmonte, driesco]@unsl.edu.ar \\ ${ }^{3}$ Department of Computer Science and Software Engineering \\ Miami University - Oxford, OH, USA \\ ahmede@miamiOH.edu
}

\begin{abstract}
Nowadays, business companies/organizations/enterprises are moving theirs processes to the cloud, on the other hand, they do not want to depend on a unique supplier and be tied to it. On the other hand, they want a variety of company that offers different technologies. But the fact that each supplier uses a different technology, make the selection a costly task that's consume a lot of time. This does not mean that the amount of suppliers should be reduced, because this is good for the market. Because of this, it is necessary to have an environment that allows the diversity and compatibility of technologies. The cloud should be thought free of incompatibilities to focus on the interoperability among the different suppliers. In other words, each supplier should open the cloud to its competitors. Given them a set of standards and rules that allows the interconnection among the products provided. In this way, the costumers would have the possibility of choosing the most adequate product for its needs and do not be limited to a particular technology. In this sense, a strategy consists on a quality models, metrics and indicators that complement the framework currently applied to the cloud migration, is proposed.
\end{abstract}

Keywords-Workflow; Workflow Management Systems, Business Process, Metrics, Cloud Computing; Performance Indicators, Evaluation Strategies. 


\section{Introduction}

Analyzing the business process life cycle [1], is highly important to achieve a continuous improvement. This makes the organizations searching for tools that give the necessary support to make these improvements. On the other hand, due of information globalization, organizations tend to virtualize their business: move their business to The Cloud. From the business process complexity point of view, one of the technologies more significant to support its automation is the Workflows Management System (WfMS). The WfMS gives computational support to define, synchronize and execute the activities of a process. To facilitate and provide flexibility to those systems, it is fundamental to have tools for measuring its quality. One of the fundamental aspects in any workflow process is having means that allow measure its most relevant elements for detecting areas and aspects of the process to be improved. For this, it is necessary to provide a framework to make this measuring. Due to this needs and following the methodology proposed in [2], a set of elemental metrics was defined used as indicators for the structural complexity of the workflow process model.

As previously mentioned, companies are looking to manage their processes in the cloud. From the point of view of process management, a workflow can be controlled manually, computerized, or as a combination of both methods. A workflow scheme allows companies to organize tasks and resources through rules that make it easier to control the business processes of the company. That is to say that with a workflow process a total and absolute control of all tasks is achieved. In this sense, companies interested in migrating their business to the cloud need to maintain that control. For this reason, is essential that the workflow process is high quality.

From this perspective, a means to obtain high quality processes, easily maintainable and adaptable, is to provide quality models of these processes. This is true for any workflow process regardless of where you are staying. In particular, if the processes will be managed in the cloud, it will be very useful to have tools to evaluate the quality of these processes.

As mentioned, a workflow process is usually a combination of computerized and manual processes. From this perspective, the question arises: is it possible or not to upload all processes to the cloud? If it is not possible, the need to determine which of these processes move and which not? It is also valid to ask if any Internet supplier in the cloud is the same. If different suppliers are chosen, is it easy to interact with the services and products they provide?

Under the previous considerations, in the field of the present research, consisting in the definition of a framework for the measurement of workflow processes that helps the improvement and maintenance of the models and the processes that they represent, it was applied This framework helps in making a decision about which processes is feasible or profitable to move to the cloud. To do this, we propose the definition of new metrics that help to this end. With the application of the metrics it is intended to show and determine the degree of the workflow process modeling helps in the continuous improvement of the business processes that you want to migrate to the cloud.

In the field of workflow processes, we can observe an important work in the modeling of these processes, thus several research lines emerge. Among these works we can highlight proposals in which the Workflows patterns are used to carry out the evaluation and/or comparison of different modeling languages $[3,4,5]$. From another point of view, in the field of measurement, various works can be observed in the measurement of business processes as in $[6,7]$. However, despite of the importance of measuring the quality of the workflow processes, it is very little the work detected in terms of the quality measurement of these processes and the models that represent them. Among the works in this field can be highlighted the proposals made in $[8,9,10]$, which proposes a metric for the measurement of the complexity of the flow of control based on the complexity cyclomatic of McCabe. Other work that can be mentioned in this field is the proposal introduced in [11,12]. In these works, the authors introduce metrics to evaluate the internal cohesion of the activities in a workflow process and the link between their activities. These proposals present alternatives for the measurement of some aspects of process modeling workflows. However, they do not cover all aspects relevant to 
them. Under these considerations, a set of metrics was defined for the measurement of the quality of workflow process models from the standpoint of their maintainability [13]. The proposed metrics help to evaluate, compare and improve the workflow models and, consequently, the processes they represent. In this context, study cases were carried out applying these metrics for the evaluation and comparison of modeling languages $[14,15]$.

With regard to cloud computing, there are currently a variety of tools and frameworks that allow workflow processes to work in the cloud. In this field, this line of research is working in a framework that helps to measure different characteristics of these processes through the proposal of a set of metrics for the individual measurement of each aspect relevant to the modeling, as well as the overall structural complexity of the process, and the models that represent it. The objective of these metrics is to provide a measurement of the structural complexity of the workflow processes and the relationship with their environment.

Because any business process execution has an underlying workflow, and the growing cloud work, this framework was applied to evaluate workflow processes in the cloud. In addition to the processes, the services of cloud computing that supported them were analyzed, because their quality is directly influenced by the quality of these services. In this context, the metrics proposed in the evaluation of the workflow processes of a medium company were applied, which aims to move their processes to the cloud, in order to increase their competitiveness. At this point arises the need to expand the proposed framework in what was called a strategy to analyze, study, measure and decide on the necessary interoperability that Internet suppliers should provide in the cloud, in any migration process to the cloud. To this end, it was necessary to define a quality model based on quality standards together with a new set of metrics and indicators that emerged from the new domain: the cloud.

\section{The Workflow Processes in the Cloud}

Cloud Computing can be thought of as a model of fast sourcing of IT resources that enhances the provision of services, business and IT, facilitating the work of the end user and the service supplier. It describes a new model of supplementing, consuming, and providing Internet protocol-based on IT services, which typically consists of provisioning dynamically scalable resources. It often takes the form of Web-based tools or applications, which users can access and use through a Web browser as if they were locally installed programs on their own computers.

To achieve their goal, Cloud Computing suppliers provide online business applications that are accessed from another WEB service or software as a Web browser, while software and data are stored on servers. In this new information management model, "sensitive" business data do not reside in enterprise facilities, which could result in a high-vulnerability context for information theft. From the point of view of business process management, organizations begin to adapt to this new trend and need to "move their business to the cloud." This could give them more market competitiveness. However, due to the structure and relationships that can be given in a business process, it is not always possible to bring the entire business to the cloud. In addition, some processes, which have some degree of communication, may be implemented and managed by different suppliers of cloud computing, which brings a new problem. In this situation, it will be necessary to determine the degree of coupling of the activities lodged in different clouds. This is important from different perspectives, such as the cost of communication, security and information protection.

This leads companies to have to conduct a study and analysis of their processes to determine which of them are feasible to migrate to the cloud. For this it is necessary to have some means to be able to carry out this evaluation. In this context, the proposed metrics were adapted and new metrics were defined that allow measuring the characteristics of the business processes and those inherent to the cloud, such as communication between processes hosted in different clouds: Is it advisable to keep 
them in separate clouds? if communication with other processes is minimal, is it advisable to upload that process to the cloud? among others.

\section{Quality and Measurement}

Software applications or products, when released to the market, are expected to have some degree of acceptance among end-users. That degree will depend on the particular characteristics that each one of them considers important when evaluating. From the point of view of Software Engineering, quality is one of the main features that an application has to have to be successful. It is relevant for software developers to be able to measure the quality or test it to the built applications, being an indispensable requirement to be able to measure, to know what is to be measured and how.

The quality of software, in the most general sense, is defined as: "Effective software process that is applied in a way that creates a useful product that provides measurable value to those who produce it and to those who use it" [16, 17]. It is also a complex combination of factors, varying between different applications. Authors like Pressman [18], McCall [19] and standards like ISO 9126 [20], ISO 14598 [21], ISO 25000 [22], among others, have tried to determine and categorize the factors that affect the quality of the software.

Thus, it is known as a model of quality to apply a set of good practices for the life cycle of the software, focused on the processes of management and development of projects [23].

Over time, different models have been developed to evaluate the quality of the software. Among the best known, can be mentioned that of McCall [19], Evans and Marciniak [24], FURPS [25], Piattini, García and Caballero [26], among others.

Related to the quality of the product, has recently appeared the set of norms ISO/IEC 25000 [22]. ISO/IEC 25000 constitutes a series of standards based on ISO/IEC 9126 [20] and en ISO/IEC 14598 [21], whose main objective is to guide the development of software products by specifying requirements and evaluating quality characteristics [22].

The quality model represents the cornerstone around which the system is established for the evaluation of the quality of the product. This model determines the quality characteristics that will be taken into account when evaluating the properties of a particular software product.

Each quality model has characteristics, subcharacteristics; metrics associated to the measurement and evaluation of the same. Thus, the product quality model defined by ISO/IEC 25010 [27] is composed of eight features: functional adequacy, performance efficiency, compatibility, usability, reliability, security, maintainability and Portability.

Metrics are a quantitative measure of the degree to which a system, component or process possesses a given attribute [28]. The indicators, on the other hand, are metrics or combination of metrics that provide a deep vision, of the process, of the project or of the product software [29]. Both elements are key assets of an organization that provide useful data and information for the analysis processes [30]. Also, they are a good means to understand, monitor, control, predict and test the development of software and maintenance projects [31].

ISO/IEC 17788 [32], defines Cloud Computing as the paradigm to allow access to the network to a scalable and elastic set of physical or virtual resources that can be shared with self-service supply and administration on demand (ISO/IEC JTC1 SC38, 2014 [33]). Another definition, widely accepted, is that provided by NIST, which defines it as a model to enable ubiquitous, convenient and ondemand network access to a shared set of configurable computing resources (e.g., networks, servers, storage, applications and services) that can be quickly supplied and released with a minimum management effort or interaction with the service supplier [34].

An outstanding aspect of Cloud Computing, is its strong focus on service orientation [35]. Currently, cloud platforms group their services into three types of provision: Infrastructure as a 
Service (IaaS), Platform as a Service (PaaS), or Software as a Service (Software as a Service, SaaS). This makes the generic quality model proposed by ISO/IEC 25010 [36] for the evaluation of software products, need to be personalized and extended, in such a way as to provide mechanisms to evaluate the specific characteristics of the Cloud services.

In recent years, some quality models have been proposed for cloud services, such as $[37,38,39$, 40], but none of these models has a complete collection of quality attributes with their respective metrics that allow to evaluate different Categories of services (SaaS, PaaS, IaaS) and cloud artifacts (cloud architecture, service in use) at different stages of the life cycle, and which in turn is aligned with ISO/IEC 25010 [27]. Some previous work has focused on the description of quality and metric characteristics to measure the quality of a cloud service from a multidimensional perspective. An example of this is the Service Measurement Index (SMI) [41], a proposed framework for comparing services or vendors with respect to six key performance indicators (KPIs): Quality, agility, risk, capacity, cost, and security. The model raised by SMI integrates more than 40 individual features related to cloud services. However, the proposal does not provide metrics to measure all the features and attributes proposed.

A model with quality attributes for the distribution of products and services in different clouds, of the different suppliers of the market, was not found for in-depth study of interoperability. In the systematic search for metrics to measure, analyze and study this particular problem, it was necessary to adapt or redefine the quality models/workflow/metric models and indicators to address this particular case. Thus, the present work proposes a model for the evaluation of the quality in the interoperability of Web services.

\section{Proposed Model}

Companies have to compete, but they have to do so in merits that actually offer added value to customers such as: reliability, consistency, security and performance or ease of use, and not on technicalities such as formats or configurations. Those things DON'T add value to customers. In this sense, there are differences between useful services, such as databases that behave differently and therefore offer different options for different cases. These differences, purely operational, establishes barriers of entry for those who want to use the cloud. It is therefore important to remove them. The following defines each of the characteristics and subcharacteristics of the proposed model:

- Compatibility

○ Coexistence

$\circ$ Interoperability

- Communication scheme or protocols

- Publication of the scheme to the public

- Schema Update time

- Standard or communication norm

- Cloud opening degree

Taking the ISO 25010 Standard [27], which is the default quality model for product quality, we can define the characteristics and subcharacteristics as follows:

Compatibility: the ability of two or more systems or components to exchange information and/or perform their required functions when sharing the same hardware or software environment. When it comes to compatibility, it takes into account two subcharacteristics that compose it and define:

1. Coexistence. Product capacity to coexist with other independent software, in a common environment, sharing common resources without detriment.

2. Interoperability. Capacity of two or more systems or components to exchange and use the information exchanged. 
The contribution to the standard 25000 , is that by extending the concept to a new paradigm such as Internet in the cloud, there are characteristics that need to be exploited or extended in attributes of the new field of application, among which are proposed: schema or protocols of communication; Publication of the scheme to the public; Schema update time; Standard or communication norm; Cloud Opening Degree.

Having a scheme that allows the exchange of data and information among different suppliers is imperative for users who want to migrate or move to the cloud, using different suppliers, or, for different reasons, operational, economic, etc., they need to have distributed the business processes in different servers/suppliers. An expected requirement would be for these schemes to be public and to be updated periodically to be up-to-date, both for communication and data exchange and information processes in terms of technology and changes in business or market. It is important that they conform to international or known standards or standards in order to save some of the Internet's own risks: such as security and impact on the environment, among others. Another desirable or expected feature is to know the degree of openness of the services, products or processes offered by the different suppliers of the cloud, that is, to know the possibility or not to connect and exchange different processes or services that are Deployed in different vendors, but that make the model of the company we want to interact with different hardware and software platforms, both own and contracted.

With the objective of studying the interoperability of the different Internet suppliers in the cloud, we proceeded to define and redefine metrics that allow quantifying each of the attributes that define each characteristic motive of the study. Once the metrics were determined, the elemental indicators were defined for the measurement of the degree of satisfaction of each characteristic evaluated; following is a summary of this.

Publication of the scheme to the public

Metric: Schema Publishing (SP): Verifying the public existence of schemas for data/information exchange.

Elemental Indicator: $\mathrm{EI}(\mathrm{SP})=\left\{\begin{array}{cc}0 & \mathrm{SP}=0 \\ 1 & \mathrm{SP}>0\end{array}\right.$

Schema Update time.

Metric: Period of publication between schemas (PPS): Time between the publications of the schemas for the exchange of data/information.

Elemental Indicator:

$$
E I(P P S)= \begin{cases}1 & P P S=0 \\ 0,5 & 0<\text { PPS }<5 \\ 0,2 & 5<=\text { PPS }<10 \\ 0 & \text { PPS }>10\end{cases}
$$

Standard or communication norm.

Metric: Degree of compliance of a standard (DCS): the degree to which the scheme follows a communication standard for data/information exchange.

Elemental Indicator: $\operatorname{El}(\mathrm{DCS})= \begin{cases}0 & \mathrm{DCS}=0 \\ 0,75 & 0<\mathrm{DCS}<2 \\ 1 & 2<=\mathrm{DCS}<7\end{cases}$

Cloud Opening degree.

Metric: Number of configurations (NofC): posting the various parameters to be configured for data/information exchange.

Elemental Indicator:

$$
\mathrm{El}(\text { NofC })= \begin{cases}1 & \text { NofC }=0 \\ 0,8 & 0<\text { NofC }<5 \\ 0,35 & 5<=\text { NofC }<10 \\ 0 & \text { NofC }>10\end{cases}
$$

Some of the subcharacteristics desirable by users of cloud services would be for the data exchange 
scheme to be available between, for example, different databases or service from different vendors to achieve better performance of Organizations ' processes, and that this scheme is updated periodically to stay optimized over time. Another characteristic is that they conform to the communication and safety standards published by some of the international quality/security agencies.

\section{Case Study}

It is an increasingly common that different companies and institutions, of any types, see the need to manage large data/information clusters. It also increases the number of critical processes managed and does not allow interruptions under any circumstances. This scenario poses a number of serious problems to these companies, including the capacity and security of their processes, which becomes increasingly difficult to manage in a local infrastructure environment. This is partly because organizations have to consider the enormous initial investment in the computer and network infrastructure that a company should have, in addition to the maintenance and technological upgrades needed over time. To confront these scenarios, although not only these, has appeared the concept of the platforms in "cloud" or "upload in the cloud". These platforms are based on the PaaS (Platform as a Service) concept, which is defined as the ability to provide a tool environment for creating applications and services that work over the Internet. Examples of these are: Amazon Web services, Microsoft Azure and Google Cloud Platform (GCP) among many others. These are the PaaS services platforms that developers and IT professionals use to create, deploy, and manage services across each vendor's global network of data processing centers. This kind of services brings robustness to the systems, offering a solution to the problems of the scenarios described above. With these platforms, hardware maintenance is not required, that is to say, the investment in infrastructures is practically null and provides mobility and security to the company, since they work online. The investment will be minimal: simply in terminals and a good connection to the Internet, in addition to manage well the tariffs of the platforms. The goal is to pay for what is used and save on local process power. Companies are now beginning to "virtualize" or "move to the cloud" many of their services and systems (Web servers, email, internal applications, etc.) as it bring new dynamism to organizations.

The work began with the choice of two database servers to store the information of the company's business processes. Then, the implementation of the business logic was hosted in a third internet supplier; it deployed a system for the collection of services and/or products sold to customers. The data/information and business processes of sales and collections of the company were uploaded to the cloud. At that moment, it did not have too many problems, but the problem of having divided the database in terms of sales on the one hand and the collections on the other, perhaps a decision not entirely accurate and correct, and when deploying the application that worked with BAS It is of data, it showed the problem that now we had the difficulty that the formats of communication or exchange of data were not transparent or friendly between them. This problem is reflected in figure 1 .

With this problem, we proceeded to look for a tool that would allow attacking it. At this point we proceeded to define a quality model that would allow studying the point of communication of the suppliers of the cloud. The main objective was to study the interoperability of the cloud. To this end, the model was instantiated quality has been previously defined. In this case the interoperability feature was assessed, key point in the decision making in the migration of the business to the cloud.

The study focused on the subcharacteristics protocols or communication scheme, i.e. the scheme of exchange of data/information/knowledge between the services of the different suppliers, and the standard or norm of communication adopted, which measures the degree of exchange pursued by the different suppliers of cloud services.

In applying the defined metrics and indicators, it was observed that none of the suppliers focused on the publication of the data and information schemes with the aim of making the exchange of 
information fluent between the different applications and services but, On the contrary, they focused only on technicalities such as formats or configurations. Which made it really little scalable and integrated the migration to the cloud.

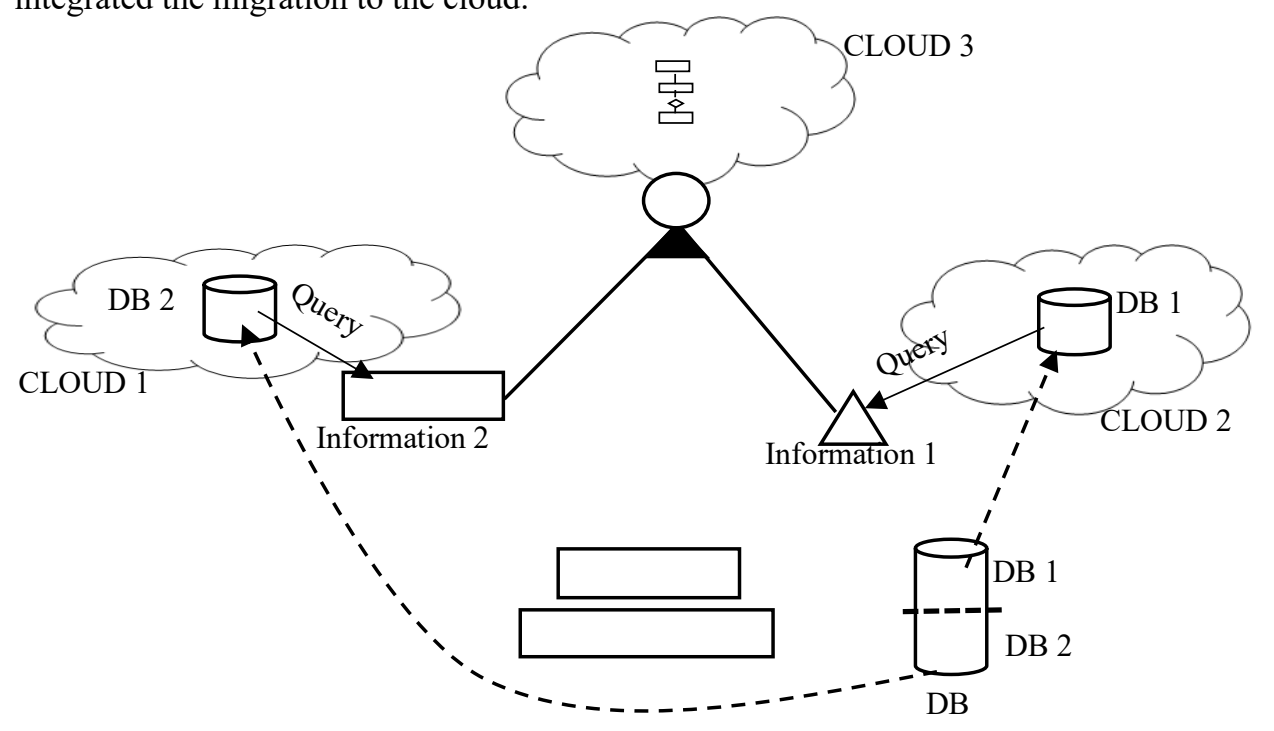

Format or structure of information of Cloud 1.

$\wedge$ rormat or structure of information of Cloud 2.

Format or structure of information of Cloud 3.

Figure 1 Distribution of processes and DB in the cloud.

This was reflected in, for example, EI (SP (CLOUD 1)): 0, EI (SP (CLOUD 2)): 0, EI (SP (CLOUD 3)): 0 , since none of the CLOUD services contracted publicly their schemes for the exchange of data/information; While the EI indicator (PPS) could not be evaluated because, when the schemas were not published, it was not possible to access the updating times of the same, so this indicator was evaluated to 0 for all services.

Consequently, of the measurements arises, a high degree of incompatibilities between the different suppliers. This situation must be left behind and generate instruments that allow at least to reduce these incompatibilities, and to move towards interoperability between the different suppliers of cloud in pursuit of the benefit of all the customers. It is clear that this situation is not simple, due in principle to strong economic/commercial competition, in particular, between suppliers of the same type of service. However, we believe that advancing in this direction will provide important benefits, not only to customers, but also to suppliers, as companies, according to their needs, will opt for those suppliers that provide them with greater interoperability with All the services that they use or need

\section{Conclusions}

Nowadays, dynamism and globalization leads companies to migrate their business processes to the cloud. However, because of the diversity of business and issues such as information security and confidentiality, companies do not want to rely on a single supplier and be fully tied to it, on the contrary, they want to diversify companies to buy their technology and services. In this sense, the fact that every supplier at the moment uses a different technology makes this a costly and time-consuming task. From this point of view, companies have to compete, but they have to do this, in order to they really offer added value to customers such as reliability, consistency, security, performance or ease of use, and not on technicalities such as formats or configurations. These things DON'T add value to 
customers

This research is based on the identification of this problem in the context of today's companies that want to move their business processes to the cloud. Once described the problem to be solved we proceeded to carry out an analysis, study and discussion of models of quality evaluation, standards and methodologies because of this, the model contemplates the totality of the characteristics to validate in the services involved. The study focused on the subcharacteristic protocols or communication scheme, i.e. the data/information/knowledge exchange scheme between the services of the different suppliers. And the standard or norm of communication adopted that measures the degree of exchange pursued by the different suppliers of cloud services.

There are differences between useful services, such as databases that behave differently and therefore offer different options for different cases. These differences create serious problems for companies that want to use these services but do not want to rely on a single supplier. Therefore, it is considered of paramount importance, to eliminate the differences, purely operative, that suppose barriers of entry for those who want to use the cloud. In this sense, a model is proposed that allows evaluating the degree of interoperability of the Web services based on different quality standards, together with a set of metrics and indicators to be able to realize the necessary measurements and calibrations to each Reality.

The proposed model was applied in a case study of a medium company that wants to move its processes to the cloud. As a conclusion of this task, the distribution of data and information on different servers with varying degrees of operability over data/information had to be reconsidered for the moment due to the inability to do collaborative work among the clouds, as the obstacles to the configurations frustrated this task and revealed the little aperture of the cloud at the moment.

\section{References}

[1] D. Georgakopoulos, A. Tsalgatidou, "Technology and Tools for Comprehensive Business Process Lifecycle Management”, Workflow Management Systems and Interoperability. Springer V. 1998.

[2] M. Serrano, M. Piattini, C. Calero, M. Genero, and D. Miranda, "Un método para la definición de métricas de software.", in 1er MIFISIS, 2002, pp. 65-74.

[3] S. A. White, "Process Modeling Notations and Workflow Patterns", in Workflow Handbook 2004, L. Fischer, Ed., ed: Published in association with the WfMC, 2004.

[4] P. Wohed, W. M. P. van der Aalst, M. Dumas, A. H.M. ter Hofstede, and N. Russell, "Pattern-based Analysis of UML Activity Diagrams", 2004.

[5] P. Wohed, W. M. P. van der Aalst, M. Dumas, A. H. M. ter Hofstede, and N. Russell, "On the Suitability of BPMN for Business Process Modelling", in 4th International Conference on Business Process Management (BPM 2006), LNCS., Vienna, Austria, 2006, pp. 161-176.

[6] E. Rolon, F. Ruiz, F. Ó. Garcia Rubio, and M. Piattini, "Aplicación de Métricas Software en la Evaluación de Modelos de Procesos de Negocio", Revista Electrónica de la Sociedad Chilena de Ciencia de la Computación, 2005.

[7] E. Rolon, F. Ó. Garcia Rubio, F. Ruiz, and M. Piattini, "Validating a Set of Measures for Business Process Models Usability and Maintainability", 2006.

[8] J. Cardoso, "How to measure the control-flow complexity of web processes and workflows", The Workflow Handbook, pp. 199-212, 2005.

[9] J. Cardoso, "Control-flow Complexity Measurement of Processes and Weyuker's Properties", 2005.

[10] J. Cardoso, "Approaches to Compute Workflows Complexity", in Dagstuhl Seminar, The Role of Business Processes in Service Oriented Architectures, Dagstuhl, germany, 2006.

[11] H. A. Reijers, "A Cohesion Metric for the Definition of Activities in a Workflow Process.", Eighth CAiSE/IFIP8.1 International Workshop on Evaluation of Modeling Methods in Systems Analysis and Design 2003, pp. 116-125, 2003.

[12] H. A. Reijers and I. T. P. Vanderfeesten, "Cohesion and Coupling Metrics for Workflow Process 
Design”, BPM 2004, LNCS 3080, pp. 290-305, 2004.

[13] M. Peralta, F. Ó. Garcia Rubio, D. Riesco, C. Salgado, and G. Montejano, "Un Conjunto de Medidas para la Evaluación de Modelos Workflow", CACIC'08, 2008.

[14] M. Peralta, F. García, M. Piattini, and R. Uzal, "Un experimento Comparativo de dos Lenguajes de Modelado Workflow: YAWL vs Diagramas de Actividad", 8th ASSE, pp. 145-154, 2007.

[15] N. Debnath, M. Peralta, C. Salgado, G. Montejano, D. Riesco, and M. Berón, "Metrics for Evaluation of Workflow Models: An Experiment for Validation", in 20th SEDE, USA, 2011.

[16] U. I. d. Telecomunicaciones, " Grupo Temático sobre ciudades sostenibles e inteligentes", 2014.

[17] M. Bouskela, M. Casseb, S. Bassi, C. De Luca, and M. Facchina, "La ruta hacia las Smart cities: Migrando de una gestión tradicional a la ciudad inteligente", 2016.

[18] R. Pressman, Ingeniería de Software. $6^{a} E d:$, 2005.

[19] J. A. McCall, P. K. Richards, and G. F. Walters, "Factors in Software Quality", RADC TR-77-369, Vols I, II, III, US Rome Air Development Center Reports NTIS AD/A-049., 1977.

[20] ISO, "ISO/IEC 9126-1:2001. Software Engineering - Software Product Quality -Part 1: Quality Model, Int'l Org. For Standardization, Geneva", ed, 2001.

[21] ISO, "ISO/IEC 14598-1. Information Technology-Software Product Evaluation - Part 1: General overview", ed, 1999.

[22] ISO, "ISO/IEC 25000. SQuaRE - System and Software Quality Requirements and Evaluation. http://iso25000.com".

[23] EcuRed, "Modelo de calidad: breves consideraciones. https://www.ecured.cu/Modelo_de_calidad. La Habana, Cuba", 2005.

[24] M. W. Evans and J. J. Marciniak, Software Quality Assurance and Management: Wiley, 1987.

[25] R. C. Grady, Deborah., Software Metrics: Establishing a Company-wide Program: Prentice Hall. Hewlett-Packard (HP), 1987.

[26] M. Piattini, F. García, and I. Caballero, Calidad de los Sistemas Informáticos: Ed. Alfaomega, 2007.

[27] ISO, "ISO/IEC 25010:2011", 2011.

[28] IEEE, "Software Engineering Standards, Standard 610.12-1990, “, ed, 1993.

[29] B. Ragland, Measure, Metric or Indicator: What is the Difference? : Ed: Crosstalk, 1995.

[30] L. Olsina, E. Pesotskaya, G. Covella, and A. Dieser, " Bridging the Gap between Security/Risk Assessment and Quality Evaluation Methods", in ASSE, 2012-2013.

[31] I. Sommerville, Ingeniería de Software. Séptima Edición: Ed: PEARSON Addison Wesley, 2005.

[32] ISO, "ISO/IEC 17788:2014 Information technology. Cloud computing. Overview and vocabulary".

[33] ISO, "ISO/IEC JTC 1/SC 38 Cloud Computing and Distributed Platforms", ed, 2014.

[34] P. M. Mell and T. Grance, "The NIST Definition of Cloud Computing. Recommendations of the National Institute of Standards and Technology", Special Publication 800-145. National Institute of Standards and Technology. U.S. Department of Commerce, 2011.

[35] J. Rhoton and R. Haukioja, Cloud Computing Architected: Solution Design Handbook, 2013.

[36] ISO, "ISO/IEC JTC 1/SC 7 Software and systems engineering", ed, 2011.

[37] X. Zheng, P. Martin, K. Brohman, and L. D. Xu, "Cloud Service Negotiation in Internet of Things Environment: A Mixed Approach", IEEE Transactions on Industrial Informatics, 2014.

[38] J. Y. Lee, J. W. Lee, D. W. Cheun, and S. D. Kim, "A Quality Model for Evaluating Software-as-aService in Cloud Computing", Software Engineering Research, Management and Applications, 2009. SERA '09. 7th ACIS International Conference, pp. 261-266, 2009.

[39] P. X. Wen and L. Dong, "Quality Model for Evaluating SaaS Service", Emerging Intelligent Data and Web Technologies (EIDWT), Fourth International Conference, pp. 83-87, 2013.

[40] P. Zhou, Z. Wang, W. Li, and N. Jiang, "Quality Model of Cloud Service. “, High Performance Computing and Communications (HPCC), IEEE 7th International Symposium on Cyberspace Safety and Security (CSS), IEEE 12th International Conferen on Embedded Software and Systems (ICESS), IEEE 17th International Conference, pp. 1418-1423, 2015.

[41] CSMIC, "Service Measurement Index (SMI) Framework", Carnegie Mellon University Sillicon Valley, Moffett Field, CA Usa 2014. 